OPEN ACCESS

Edited by:

Hongmin Cai,

South China University of Technology,

China

Reviewed by:

Wei-Hua Chen,

Huazhong University of Science and

Technology, China

Wen Zhang,

Huazhong Agricultural University,

China

*Correspondence:

Yuanyuan Ma

chonghua_1983@126.com

Specialty section:

This article was submitted to

Bioinformatics and

Computational Biology,

a section of the journal

Frontiers in Genetics

Received: 22 August 2019

Accepted: 24 January 2020

Published: 21 February 2020

Citation:

Ma Y, Liu G, Ma Y and Chen Q (2020) Integrative Analysis for Identifying Co-

Modules of Microbe-Disease Data by

Matrix Tri-Factorization With

Phylogenetic Information.

Front. Genet. 11:83

doi: 10.3389/fgene.2020.00083

\section{Integrative Analysis for Identifying Co-Modules of Microbe-Disease Data by Matrix Tri-Factorization With Phylogenetic Information}

\author{
Yuanyuan $\mathrm{Ma}^{1 *}$, Guoying Liu ${ }^{1}$, Yingjun $\mathrm{Ma}^{2}$ and Qianjun $\mathrm{Chen}^{2,3}$ \\ ${ }^{1}$ School of Computer and Information Engineering, Anyang Normal University, Anyang, China, ${ }^{2}$ School of Computer, Central \\ China Normal University, Wuhan, China, ${ }^{3}$ School of Life Science, Hubei University, Wuhan, China
}

Microbe-disease association relationship mining is drawing more and more attention due to its potential in capturing disease-related microbes. Hence, it is essential to develop new tools or algorithms to study the complex pathogenic mechanism of microbe-related diseases. However, previous research studies mainly focused on the paradigm of "one disease, one microbe," rarely investigated the cooperation and associations between microbes, diseases or microbe-disease co-modules from system level. In this study, we propose a novel two-level module identifying algorithm (MDNMF) based on nonnegative matrix tri-factorization which integrates two similarity matrices (disease and microbe similarity matrices) and one microbe-disease association matrix into the objective of MDNMF. MDNMF can identify the modules from different levels and reveal the connections between these modules. In order to improve the efficiency and effectiveness of MDNMF, we also introduce human symptoms-disease network and microbial phylogenetic distance into this model. Furthermore, we applied it to HMDAD dataset and compared it with two NMF-based methods to demonstrate its effectiveness. The experimental results show that MDNMF can obtain better performance in terms of enrichment index (El) and the number of significantly enriched taxon sets. This demonstrates the potential of MDNMF in capturing microbial modules that have significantly biological function implications.

Keywords: microbe-disease association, matrix factorization, phylogenetic distance, human microbiome, co-modules

\section{INTRODUCTION}

With the development of high-throughput sequencing technology, such as 16S ribosomal RNA (16S rRNA), more and more microbes were identified. Nearly $10^{14}$ bacterial cells are existed in human internal gut and provide a wide variety of gene products which induce diverse metabolic activities (Micah et al., 2007; Shah et al., 2016). The dynamic balance of human microbiome composition is essential to maintain good health. Once such balance is broken, many closely related human disease and disorders may be caused (Medzhitov, 2007; Thiele et al., 2013), such as colorectal cancer (CRC) 
(Boleij et al., 2014), obesity (Turnbaugh et al., 2009), inflammatory bowel disease (IBD) (Qin et al., 2010), bacterial vaginosis (Fredricks et al., 2005), and so on. For example, Jorth et al. have reported that gene expression profiles of periodontitisrelated microbial communities have highly conserved changes, relative to healthy samples (Jorth et al., 2014). It means that microbiome composition changes in oral cavity could be associated with pathogenesis of periodontitis. Furthermore, Socransky et al. have found that subgingival plaque is connected with several major microbial taxon including Fusobacterium, Prevotella, and so on (Socransky et al., 1998). Chen et al. have observed that the colonization with Helicobacter pylori has negative correlation with the symptom of allergy (pollens and molds), especially in the childhood (Chen and Blaser, 2007; Blaser, 2014). All these reveal the potential association between pathogenic microorganisms and complex human diseases.

Considering the key role of microbes in health, many important projects including the Human Microbiome Plan (HMP) (Gevers et al., 2012), the Earth Microbiome Project (EMP) (Gilbert et al., 2010), Metagenomics of the Human Intestinal Tract (MetaHIT) (Ehrlich and Consortium, 2011) were launched to investigate the relationships between microbiota and diseases. Moreover, some related databases and tools have been developed to analyze the increasing information for disease-related microbes. A human microbe-disease association database, called HMDAD (Ma et al., 2016a), manually collected 483 microbe-disease association entries from previously published literatures. These databases provide a possibility for microbe-disease association relationship prediction by computational approaches. Zhang et al. proposed bidirection similarity integration method (BDSILP) for predicting microbe-disease associations by integrating the disease-disease semantic similarity and the microbe-microbe functional similarity. Wang et al. proposed a semisupervised computational model called LRLSHMDA to predict large-scale microbe-disease association (Wang et al., 2017). Huang et al. combined neighbor-based collaborative filtering and graphbased model into a unified objective function to predict microbe-disease relationship (Huang et al., 2017). He et al. integrated symptom-based disease similarity network into graph regularized nonnegative matrix factorization models (GRNMF), meanwhile utilizing neighbor information to boost the performance of GRNMF (He et al., 2018). Zhang et al. utilized the advantages of ensemble learning to improve the performance of association prediction, which provided a new way for mining microbe-disease relationship (Zhang et al., 2018a; Zhang et al., 2019). All these efforts pave the way for further understanding complex regulatory mechanisms by means of which disease-related microbiota get involved.

However, cellular system is complicatedly organized and biological functions are mainly performed in a highly modular manner (Barabasi and Oltvai, 2004; Chen and Zhang, 2018). In microbial ecosystems, microbes often cooperate with each other to finish some biochemical activities. For example, ammonifiers decompose nitrogen-containing organic compounds to release ammonia. Nitrous acid bacteria (also known as ammonia oxidizing bacteria) oxidize ammonia to nitrous acid. Then, nitric acid bacteria (also known as nitrous acid oxidizing bacteria) oxidize nitrous acid to nitric acid. These two types of bacteria can obtain the energy needed for growth from the above oxidation process. Therefore, the mutualism relationship among ammonifier, nitrous acid bacteria, and nitric acid bacteria forces them to form a tight biological community. Guo et al. studied the contributions of high-order metabolic interactions to the activity of four-species microbial community and demonstrated that the interactions between pairwise species play an important role in predicting the complex cellular network behavior (Guo and Boedicker, 2016). Although knowledge about microbe-disease associations could provide helpful insights into understanding complex disease mechanisms (Huang et al., 2017; He et al., 2018), the "one-disease, many microbes" models ignore interactions within microbial community composed of several species.

Recently, multilayer interaction and modular organization have attracted more and more attentions. Several studies proposed co-module discovery methods to identify combinatorial patterns using pairwise gene expression and drug response data (Kutalik et al., 2008; Chen and Zhang, 2016). In addition, Chen et al. proposed a new method based nonnegative matrix factorization (NMF) to reveal drug-gene module connections from different molecular levels (Chen and Zhang, 2018). Cai et al. proposed a new network-guided sparse binary matching model to jointly analyze the gene-drug patterns hidden in the pharmacological and genomic datasets with the additional prior information of genes and drugs (Cai et al., 2018). Chen et al. also proposed a higher order graph matching with multiple network constraints (gene network and drug network) to identify co-modules from different multiple data sources (Chen et al., 2018).

All these have made great progresses to study the coordinate regulatory mechanisms between two or more biological molecular networks from a systematic view. However, as far as we know, less work focuses on microbe-disease co-modules discovering. Previous studies mainly aimed to microbe-disease association prediction, and did not reveal within-module interactions (microbe-microbe, disease-disease) from the same level and cross-module interactions (microbe-disease) from multiple molecular levels.

To this end, we design a new algorithm based on NMF to construct the two-level microbe-disease module network by Gaussian profile kernel similarity (MDNMF). In order to improve efficiency and effectiveness of the proposed algorithm, we introduce human symptoms-disease network (Zhou et al., 2014) and microbial phylogenetic distance into this model, which makes functionally similar microbes (diseases with similar symptoms) tend to appear in the same microbial module (disease module). We applied MDNMF to HMDAD dataset and compared it with two classical NMF methods to demonstrate its effectiveness. The experimental results show that the majority of identified microbial modules have significant functional implications [significantly enriched in taxon sets that 
refer to groups of microbes that has something in common (Dhariwal et al., 2017)]. Figure 1 gives the illustrative example of MDNMF.

The contribution of this paper lies in (1) an efficient two-level module discovering algorithm (MDNMF) has been proposed to reveal microbe-microbe, disease-disease and microbe-disease modules association. (2) The phylogenetic distance of diseaserelated microbes is introduced into the proposed MDNMF model to make phylogenetically close microbes tend to intertwine in the development of similar disease. To our knowledge, this is the first attempt to link microbial phylogenetic relatedness to NMF-based module identification. (3) The proposed MDNMF algorithm is easily extended to other multiple-level molecular network application, for example, virushost co-modules, microbe-drug co-modules discovering, and so on. The rest of this paper is organized as: in the next section, we give a brief overview of NMF and MDNMF. And then, followed by the experimental results and the conclusions are provided in the last section.

\section{MATERIALS AND METHODS}

\section{Dataset}

The dataset is downloaded from the Human Microbe-Disease Association Database (HMDAD, http://www.cuilab.cn/hmdad) (Ma et al., 2016a). It contains 483 microbe-disease associations, which cover 292 microbes and 39 diseases. By 16S RNA sequencing techniques, most microbe names was recorded at the genus level. Based on these known microbe-disease relation, an adjacency matrix $X \in \mathbb{R}^{292 \times 39}$ can be constructed where $X_{i j}=1$ if microbe $i$ is related to disease $j$, and vice versa.

\section{The NMF Model}

NMF and its variants have been widely applied to various fields including bioinformatics (Ma et al., 2016b; Ma et al., 2017; Chen and Zhang, 2018). In NMF, given an original data matrix $X \in \mathbb{R}^{n \times m}$, we seek to find two low-rank matrices $W \in \mathbb{R}^{n \times k}$ (also called basis matrix) and $H \in \mathbb{R}^{k \times m}$ (coefficient matrix) to approximate $X$, such that $X \approx W H$, where $k<<\min (m, n)$. Here, data $X$ can be represented as the linear additional combination of basis vectors. We can obtain such a decomposition by solving the following least squares problem:

$$
\min _{W, H \geq 0}\|X-W H\|_{F}^{2}
$$

where $\|\bullet\|_{F}$ denotes Frobenius norm.

\section{Gaussian Interaction Profile Kernel Similarity for Microbes}

Based on the hypothesis that functionally similar microbes could be associated with more common human diseases, Gaussian kernel interaction profiles can be used to calculate the inferred microbe similarity (Wang et al., 2017; He et al., 2018). Given microbe-disease association matrix $X$, the ith row of $X$ indicates the interaction profiles between microbe $m_{i}$ and all the diseases. For any two microbes $m_{i}$ and $m_{j}$, their similarity can be computed as follows:

$$
\operatorname{MS}\left(m_{i}, m_{j}\right)=\exp \left(-\gamma_{m}\left\|X_{i, *}-X_{j,}\right\|^{2}\right)
$$

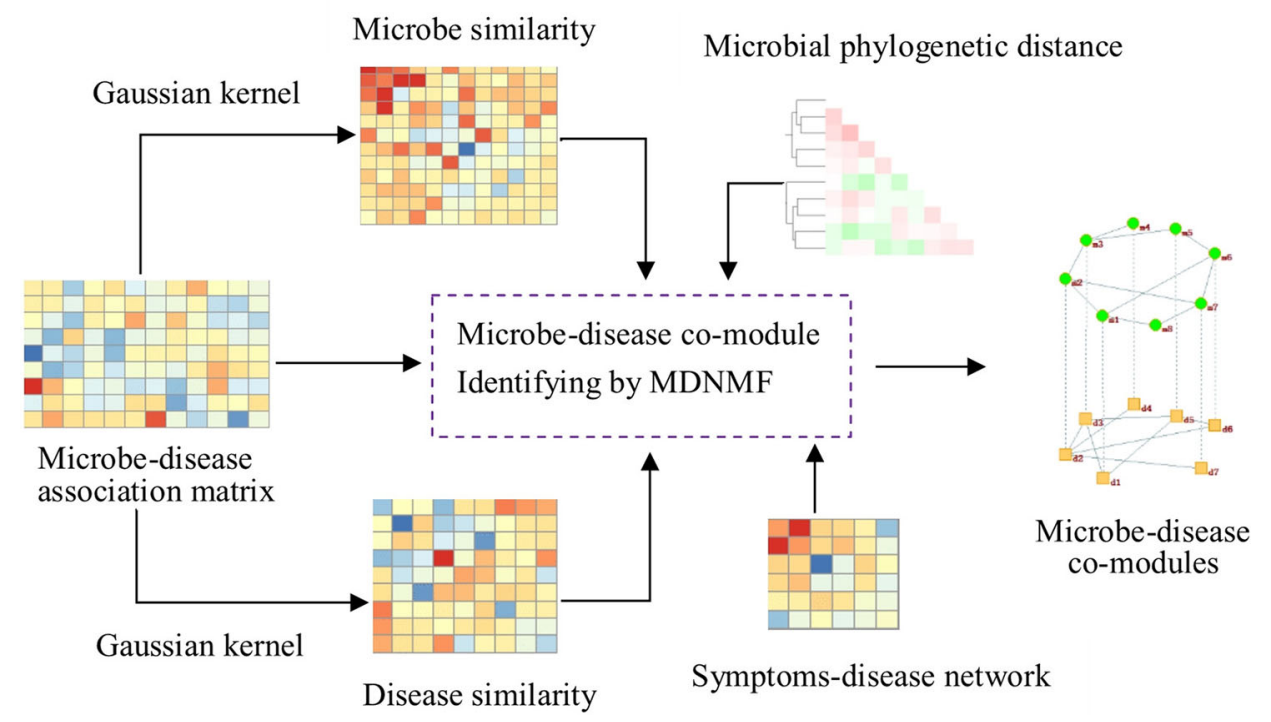

FIGURE 1 | Illustrative example of MDNMF. First, based on Gaussian kernel function we can obtain microbe and disease similarity matrices from the original microbe-disease association matrix. Then, these three matrices are served as the input of MDNMF. Simultaneously, in order to improve the accuracy of module finding and biological interpretability of modules identified by MDNMF, human symptoms-disease network and microbial phylogenetic distance are also introduced into the model. At last, microbe-disease co-modules from different levels can be obtained. 
where $X_{i, *}$ denotes the ith row of matrix $X . \gamma_{m}$ is bandwidth parameter that needs to be normalized based on a novel bandwidth parameter $\gamma_{m}^{\prime}$ and the interaction profile for each microbe, i.e., the ith row of $X$ :

$$
\gamma_{m}=\gamma_{m}^{\prime} /\left(\frac{1}{n_{m}} \sum_{i=1}^{n_{m}}\left\|X_{i,} *\right\|^{2}\right) .
$$

Here, $n_{m}$ is the number of microbes related to all diseases (here, $n_{m}=292$ ). $\gamma_{m}^{\prime}$ was set as 1 according to the previous study (Wang et al., 2017). In this way, microbe similarity matrix MS can be constructed, the element of $M S$ indicates the similarity score between two arbitrary microbes.

\section{Gaussian Interaction Profile Kernel Similarity for Diseases}

Similarly, Gaussian kernel based disease similarity matrix can be inferred as follows:

$$
\begin{gathered}
D S\left(d_{i}, d_{j}\right)=\exp \left(-\gamma_{d}\left\|X \star_{, i}-X \star_{, j}\right\|^{2}\right) \\
\gamma_{d}=\gamma_{d}^{\prime} /\left(\frac{1}{n_{d}} \sum_{i=1}^{n_{d}}\left\|X \star_{, i}\right\|^{2}\right),
\end{gathered}
$$

where $X_{*, i}$ denotes the ith column of $X, n_{d}$ is the number of diseases related to all microbes $\left(n_{d}=39\right), \gamma_{d}^{\prime}$ was also assigned to 1 .

\section{Phylogenetic Distance for Disease-Related Microbes}

Gaussian interaction profiles kernel similarity reflects the intertwining between microbes in term of microbe-disease association relationship. However, functionally similarity could not be explained only by disease relatedness, homology and phylogenetic correlation should be considered as side information to make the connected microbes in the microbe-disease association matrix likely to be placed in the same co-modules.

We searched 91 nucleotide sequences of disease-related microbes from NCBI, and imported them into MEGA to compute the phylogenetic distance between pairwise sequences by Kimura 2-parameter model. Other parameters are set in default. Thus, we can obtain the final microbial phylogenetic distance matrix $M_{p h y}$ which is used to enforce microbe members within identified modules likely to be near in phylogeny.

In order to demonstrate the role of phylogenetic information in identifying disease-related microbe modules, we extract the top 10 largest and smallest phylogenetic distance pairs as illustrative examples to further analyze whether closely related taxa tend to associate with the same disease, or similar diseases. For each microbe-microbe phylogenetic distance pair, we compute the Jaccard coefficient (JC) between two microbe-related disease profiles (rows of microbe-disease association matrix). The results shows that top 10 microbe pairs which are closely related in genetic have the largest JCs in terms of disease profile similarities. Similarly, we also compute the disease similarities between phylogenetically distant microbes and find that 9 in 10 microbe pairs have the smallest JCs. This suggests that closely related taxa tend to associate with the same disease or similar diseases, and phylogenetically distant taxa usually have distinct disease profiles.

\section{The MDNMF Algorithm}

Besides the typical NMF as Dataset described, tri-factor NMF (tri$\mathrm{NMF}, X \approx F S G$ ) is also an important matrix factorization method for clustering (Ding et al., 2006). In tri-NMF, factorized matrices $F, G$ provide an approach to perform biclustering of $X$, respectively. Factorized matrix $s$ not only provides an additional degree of freedom to enforce the reconstruct error tiny, but also implicitly denotes the relationship between clusters (Ding et al., 2005). In particular, given the symmetric similarity matrix $A$, we can decompose it into $A \approx H S_{H}{ }^{T}$. The similarity matrix reflects the intrinsic connection patterns within its original data matrix (Van Dam et al., 2017). In this paper, we propose a novel algorithm MDNMF to simultaneously factorize two similarity matrices (microbe similarity matrix $M S$, disease similarity matrix $D S$ ) and one microbe-disease association matrix $X$. The objective function is formulated as follows:

$$
\begin{gathered}
\min _{H_{1}, H_{2}, S_{1}, S_{2}}\left\|M S-H_{1} S_{1} H_{1}^{T}\right\|_{F}^{2}+\lambda_{1}\left\|X-H_{1} H_{2}^{T}\right\|_{F}^{2}+\lambda_{2} \\
\left\|D S-H_{2} S_{2} H_{2}^{T}\right\|_{F}^{2} \\
\text { s.t. } \quad H_{1}, H_{2}, S_{1}, S_{2} \geq 0 .
\end{gathered}
$$

where $M S \in \mathbb{R}^{n} m^{\times n} m, D S \in \mathbb{R}^{n} d^{\times n} d$ are microbe-microbe and disease-disease similarity matrices, respectively. $H_{1} \in \mathbb{R}^{n} m^{\times k}$, $H_{2} \in \mathbb{R}^{n} d^{\times k}$ are cluster indication matrices, $S_{1} \in \mathbb{R}^{k \times k}, S_{2} \in \mathbb{R}^{k \times k}$ are the symmetric matrices. Here, $k$ is the number of clusters, and $\lambda_{1}, \lambda_{2}$ are the parameters to balance the weights of three terms in Eq.6. The second term $\left\|X-H_{1} H_{2}^{T}\right\|_{F}^{2}$ establishes the one-to-one relationships between identified microbe modules and disease modules. Moreover, it can be regarded as a tri-NMF $\left\|X-H_{1} I H_{2}^{T}\right\|_{F}^{2}$, here $I$ is the identity matrix which enforce the $i$ th module identified by microbe clustering indication matrix $H_{1}$ is only bound up with the $i$ th module by $H_{2}$. The other two terms respectively identify one type of modules at individual levels and reveal the module associations within them via $S_{1}$ and $S_{2}$.

In order to further improve the performance of the proposed algorithm, we introduce symptoms-based disease similarity network and microbial phylogenetic distance into MDNMF. The symptoms-based disease similarity was previously studied based on co-occurrence of disease/symptom terms (Zhou et al., 2014). Here, we use $D S_{\text {sym }}$ to denote symptoms-based disease similarity matrix. The objective function of MDNMF (Eq.6) can be rewritten as follows:

$$
\begin{gathered}
\min _{H_{1}, H_{2}, S_{1}, S_{2}}\left\|M S-H_{1} S_{1} H_{1}^{T}\right\|_{F}^{2}+\lambda_{1}\left\|X-H_{1} H_{2}^{T}\right\|_{F}^{2}+\lambda_{2} \\
\left\|D S-H_{2} S_{2} H_{2}^{T}\right\|_{F}^{2}+\mu\left(\operatorname{tr}\left(H_{1}^{T} L_{1} H_{1}\right)+\operatorname{tr}\left(H_{2}^{T} L_{2} H_{2}\right)\right) \\
\text { s.t. } \quad H_{1}, H_{2}, S_{1}, S_{2} \geq 0 .
\end{gathered}
$$


Where $L_{1}=D_{1}-M S_{p h y}, L_{2}=D_{2}-D S_{\text {symp }}$ are Laplacian matrices, $\left(D_{1}\right)_{i}=\sum_{j}\left(M S_{p h y}\right)_{i j},\left(D_{2}\right)_{i}=\sum_{j}\left(D S_{s y m p}\right)_{i j}$ are degree matrices, respectively. $M S_{p h y}=1-M_{p h y} \mu$ is the regularization parameter and the whole last term in Eq.7 is used to exert a penalty for violating the prior cognition about microbial phylogeny and disease phenotype associations.

Note that disease symptoms dataset collected from PubMed literatures contains diseases and symptoms terms. The association between symptoms and diseases are quantified using term co-occurrence (just like in the field of information retrieval, if the document and keyword simultaneously appear, the corresponding position of the word-document matrix is set to the frequency of co-occurrence). And then, each disease can be represented by a vector of symptoms. At last, the cosine similarity function is used to quantify the similarity between two diseases. The link weight between two diseases quantifies the similarity of their respective symptoms. Thus, these two disease similarities based on microbes and human symptoms are different essentially in that HMDAD dataset describes the binary relationships between microbes and diseases, however, disease symptoms dataset describes the co-occurrence relationships between symptoms and diseases. Integrating them into the objective of MDNMF will simultaneously take account of the diffusion and propagation of the information from different source.

We used the multiplicative update rules to solve MDNMF problem and can find a local minimal solution by alternately updating matrices $H_{1}, H_{2}, S_{1}, S_{2}$.

(1) Fix $H_{1}, H_{2}, S_{2}$ and update $S_{1}$ with

$$
\left(S_{1}\right)_{i j} \leftarrow\left(S_{1}\right)_{i j} \frac{\left(H_{1}^{T} M S H_{1}\right)_{i j}}{\left(H_{1}^{T} H_{1} S_{1} H_{1}^{T} H_{1}\right)_{i j}}
$$

(2) Fix $H_{1}, H_{2}, S_{1}$ and update $S_{2}$ with

$$
\left(S_{2}\right)_{i j} \leftarrow\left(S_{2}\right)_{i j} \frac{\left(H_{2}^{T} D S H_{2}\right)_{i j}}{\left(H_{2}^{T} H_{2} S_{2} H_{2}^{T} H_{2}\right)_{i j}}
$$

(3) Fix $S_{1}, S_{2}, H_{2}$ and update $H_{1}$ with

$$
\left(H_{1}\right)_{i j} \leftarrow\left(H_{1}\right)_{i j} \frac{\left(2 M H_{1} S_{1}+\lambda_{1} X H_{2}+\mu D_{1} H_{1}\right)_{i j}}{\left(2 H_{1} S_{1} H_{1}^{T} H_{1} S_{1}+\lambda_{1} H_{1} H_{2}^{T} H_{2}+\mu M S_{p h y} H_{1}\right)_{i j}}
$$

(4) Fix $S_{1}, S_{2}, H_{1}$ and update $H_{2}$ with

$$
\left(H_{2}\right)_{i j} \leftarrow\left(H_{2}\right)_{i j} \frac{\left(2 \lambda_{2} D S H_{2} S_{2}+\lambda_{1} X^{T} H_{1}+\mu D_{2} H_{2}\right)_{i j}}{\left(2 \lambda_{2} H_{2} S_{2} H_{2}^{T} H_{2} S_{2}+\lambda_{1} H_{2} H_{1}^{T} H_{1}+\mu D S_{s y m p} H_{2}\right)_{i j}}
$$

\section{Determination of Modules}

In fact, the same microbe may play different roles in the development of diseases. Therefore, the idea of soft clustering is more suitable to model the function associations among microbes. The factorized matrices $H_{1}, H_{2}$ can be used to identify two types of modules, respectively. The elements with relatively large values of each column of $H_{1}\left(H_{2}\right)$ is assigned to the members of corresponding module. We calculate the threshold for each feature (each row $h_{i, \star}^{1}$ of $H_{1}\left(h_{i, \star}^{2}\right.$ of $\left.H_{2}\right)$ ) with

$$
T h(f)=\mu(f)+t \sigma(f)
$$

where $\mu(f)=\frac{1}{k} \sum_{k} h_{f k}, \sigma(f)=\sqrt{\frac{1}{n-1} \sum_{k}\left(h_{f k}-\mu(f)\right)^{2}}, t$ is a given threshold. Based on this rule, we determined the ith module members if the entries of $h_{f i}^{*}$ are larger than Th $(f)$. In Experimental Results and Discussion section, we set $t=1.5$ for two clustering indication matrices $H_{1}$ and $H_{2}$ to identify modules with proper resolution.

\section{Determination of Module Links}

Given the symmetric similarity matrix $A$, tri-NMF factorizes it to be $A \approx H S H^{T}=\sum_{i=1}^{k} \sum_{j=1}^{k} s_{i j} h_{i} h_{j}^{T}$. Here, $h_{i}$ denotes the $i t h$ column of $H, s_{i j}$ is the corresponding element of $s$. The latent clustering indication vector $h_{i}$ can reconstruct the original similarity matrix $A$, and $s_{i j}$ can be viewed as the weight of $h_{i} h_{j}^{T}$ . It means that the larger $s_{i j}$ is, the stronger the connection between the modules identified by $h_{i}$ and $h_{j}$ is. Therefore, the diagonal elements of $s$ can be used to evaluate the quality of clustering, and the off-diagonal elements can be used to establish the possible connections between different modules.

\section{Functional Enrichment Analysis for Co-Modules}

We use MicrobiomeAnalyst (Dhariwal et al., 2017) tools to conduct functional enrichment analysis for microbe modules, and select the significantly enriched taxon set terms if $P$-value $<$ 0.005 and FDR $<0.05$ (hypergeometric tests). Because MicrobiomeAnalyst provides 229 taxon sets associated with host-intrinsic factors such as diseases. For microbe-disease comodules we define the enrichment indices between significantly enriched taxon set terms and diseases within the same co-module to evaluate the performance of different algorithms. The enrichment index (EI) is formulated as follows:

$$
E I=\frac{\mid\{\text { significantly enriched taxon } \text { set }\} \cap\{\text { diseases }\} \mid}{\mid\{\text { significantly enriched taxon } \text { set }\} \cup\{\text { diseases }\} \mid},
$$

where $\mid\{$ significantly enriched taxon set $\} \mid$ denotes the number of significantly enriched taxon sets, $\mid\{$ diseases $\} \mid$ denotes the number of diseases which is related to microbes within the same comodule. Generally speaking, higher $E I_{s}$ indicates good clustering quality of identified co-modules.

\section{EXPERIMENTAL RESULTS AND DISCUSSION}

\section{Results and Comparison}

We compared MDNMF with typical NMF and NetNMF (Chen and Zhang, 2018) (without considering microbial phylogenetic 
information and symptoms-based disease similarity) by applying them to HMDAD dataset. Since NMF-based algorithms cannot guarantee a global optimal solution, we run 50 times with different initializations and selected the factorization with minimal objective function value as the downstream analysis.

We adopted EI (as described in Functional Enrichment Analysis for Co-Modules) and the number of significantly enriched microbe taxon set $\left(T S_{\text {sig }}\right)$ as metrics to evaluate the performance of different algorithms. Other taxon sets (OTS=| $\{$ significantly enriched taxon set $\}|=|$ identified disease-related taxon sets|) indicate the significantly enriched taxon sets that are not considered by EI. To some extent, the number of other taxon sets reflects the identified ability of different methods in potential microbe function modules discovering. Extensive

TABLE 1 | The performance of three co-model discovering algorithms in term of $E l$ and $T S_{\text {sig. }}$.

\begin{tabular}{lcccc}
\hline & (\#) identified co-modules & EI & (\#) $\boldsymbol{T S}_{\text {sig }}$ & OTS \\
\hline NMF & 12 & 0.08676 & 39 & 29 \\
NetNMF & 13 & 0.11563 & 49 & 36 \\
MDNMF & 14 & 0.30182 & 62 & 48
\end{tabular}

*(P-value $<0.005$ and FDR $<0.05)$. \# represents the number of identified co-modules or significantly enriched taxon sets. comparison experiments are conducted and the results are shown in Table 1.

As Table 1 shown, compared with other two NMF-based algorithms, MDNMF achieves the best performance in terms of $E I$ and $T S_{\text {sig, }}$, indicating that MDNMF could potentially discover the meaningful function modules as much as possible by introducing symptoms-based disease network and microbe phylogenetic distance.

\section{Comparison of All the Significantly Enriched Taxon Sets of Modules Identified by MDNMF, NMF, and NetNMF}

To demonstrate the effectiveness of MDNMF, we compared the microbe modules identified by these three approaches in terms of biologically functional enrichment. We performed microbe taxon set enrichment analysis for these three groups of modules and reserved the taxon set $(T S)$ terms (FDR $<0.05$, hypergeometric test) which are significantly enriched by two modules derived of MDNMF and NetNMF (or NMF). Then, for each TS term, we calculated enrichment scores $(-\log 10(p$ value)) and took the highest scores among all modules as the final score of this TS for each method. Note that the co-modules identified by MDNMF cover about 20 microbes and 3 diseases on average. There is only one co-module which contains no

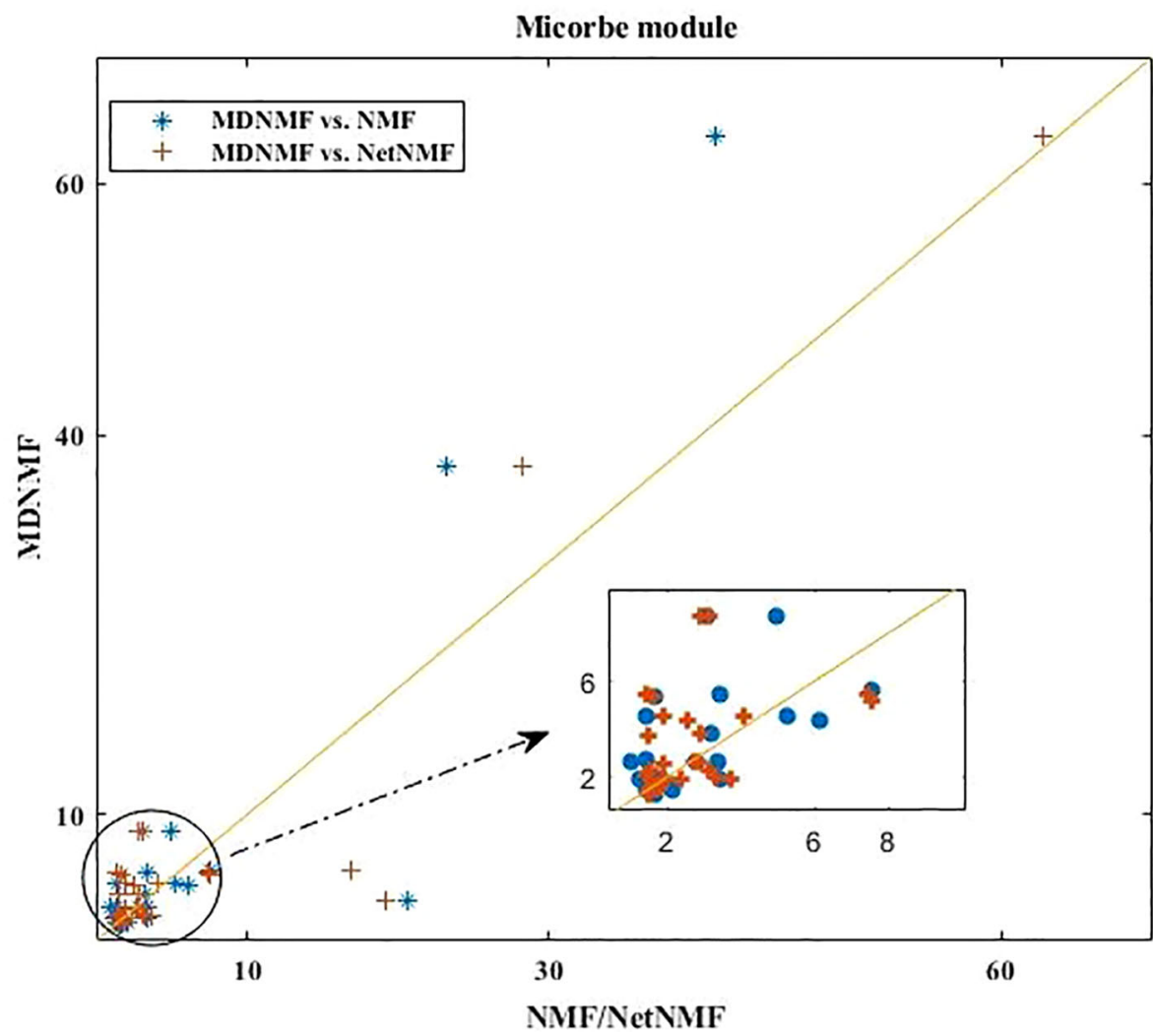

FIGURE 2 | Comparison of all the enriched TS terms of microbe modules detected by MDNMF, NMF, and NetNMF using HMDAD dataset. 
diseases. This is consistent with the average size of each microbe or disease module (see Parameter Analysis).

Applying MDNMF to HMDAD dataset, many TS terms are above the diagonal line (see Figure 2). Specifically, the enriched TS terms obtained by MDNMF have more significant Q-value $($ FDR $<0.05)$ than those of NMF and NetNMF. For microbe modules, 58.33\% (MDNMF versus NMF, $\mathrm{P}<0.005$ and FDR $<$ 0.05 , hypergeometric test) and $47.06 \%$ (MDNMF versus NetNMF, $\mathrm{P}<0.005$ and FDR $<0.05$, hypergeometric test $)$ TS terms are above the central diagonal line, respectively.

As Figure 2 shown, compared to NetNMF, microbe modules identified by MDNMF had lower significance for $52.94 \%$ modules. One of the possible reasons is that when selecting microbes, NetNMF just concerns the relationships among microbes from the original microbe-disease association matrix, whereas MDNMF has to take their phylogenetic relationships into account. This kind of extra constrains of MDNMF might affect the selected microbe subsets and their enriched functions. Despite that, MDNMF still identified more significantly enriched taxon sets than NetNMF (62 vs. 49, Table 1).

\section{Parameter Analysis}

In MDNMF, there are three parameters: $\lambda_{1}, \lambda_{2}$ ' and $\mu$. We set $\lambda_{1}=\frac{n_{m}}{n_{d}}, \lambda_{1}=\frac{n_{m}^{2}}{n_{d}^{2}}$ according to the previous study (Chen and Zhang, 2018). When applying these three NMF-based algorithms to HMDAD data, the reduced dimension $k$ is needed to be predetermined. Here, we selected $k=15$ from the candidate set $\{10,15,20\}$, and $\mu=0.001$ from $\{0.001,0.01,0.1\}$, respectively. Under this setting, the number of identified microbe modules with significantly enriched taxon sets terms is highest (hypergeometric tests, $\mathrm{P}$-value $<0.005$ and FDR $<0.05$ ). Mode selection is demonstrated in Figure 3.

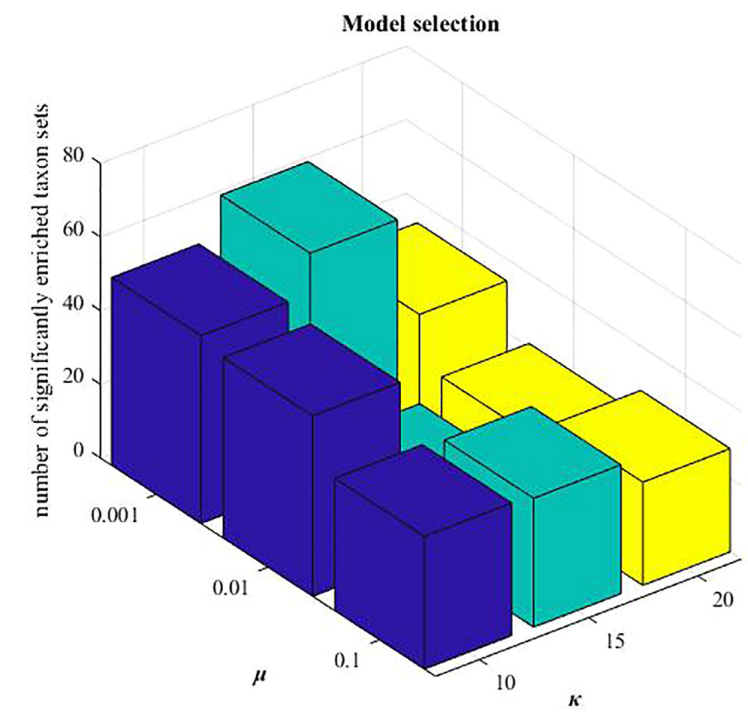

FIGURE 3 | Model selection of parameters: $\mu$ and $k$.

\section{Case Studies}

To further validate the performance of MDNMF, we select several microbe-disease co-modules identified by MDNMF to analyze their biological functions and inner connections. In total, $60 \%$ microbe modules are enriched in at least one TS term. In these identified microbe-disease co-modules, the diseases caused

TABLE 2 | The identified microbe-disease co-modules by MDNMF.

\begin{tabular}{|c|c|c|c|c|}
\hline $\begin{array}{l}\text { Co- } \\
\text { module_id }\end{array}$ & $\begin{array}{l}\text { Disease } \\
\text { module }\end{array}$ & $\begin{array}{l}\text { Microbe } \\
\text { module }\end{array}$ & $\begin{array}{c}\text { Taxon sets } \\
\text { (matched disease, } \\
\text { descending order } \\
\text { by FDR) }\end{array}$ & $\begin{array}{l}\text { Associated } \\
\text { co-module }\end{array}$ \\
\hline 9 & $\begin{array}{l}\text { Bacterial } \\
\text { Vaginosis } \\
\text { Clostridium } \\
\text { difficile } \\
\text { infection } \\
\text { (CDI) } \\
\text { Ileal } \\
\text { Crohn's } \\
\text { disease(CD) } \\
\text { Irritable } \\
\text { bowel } \\
\text { syndrome } \\
\text { (IBS) } \\
\text { Liver } \\
\text { cirrhosis } \\
\text { Necrotizing } \\
\text { Enterocolitis } \\
\text { Periodontal } \\
\text { Type } 1 \\
\text { diabetes }\end{array}$ & $\begin{array}{l}\text { Actinobacteria } \\
\text { Bacteroidaceae } \\
\text { Bacteroides } \\
\text { Bacteroides } \\
\text { uniformis } \\
\text { Bacteroidetes } \\
\text { Firmicutes } \\
\text { Fusobacteria } \\
\text { Fusobacterium } \\
\text { Haemophilus } \\
\text { Lachnospiraceae } \\
\text { Lactobacillus } \\
\text { Prevotella } \\
\text { Proteobacteria } \\
\text { Streptococcus } \\
\text { Veillonella }\end{array}$ & $\begin{array}{l}\text { Liver Cirrhosis } \\
\text { Chronic Obstructive } \\
\text { Pulmonary Disease } \\
\text { Bacterial Vaginosis } \\
\text { (increase) } \\
\text { Asthma } \\
\text { Colorectal } \\
\text { Carcinoma } \\
\text { Resistance to } \\
\text { Immune } \\
\text { Checkpoint } \\
\text { Inhibitors (increase) } \\
\text { Type I Diabetes } \\
\text { Diarrhea Irritable } \\
\text { Bowel Syndrome } \\
\text { (IBS) } \\
\text { Parkinsons } \\
\text { (increased) } \\
\text { Third Trimester (vs } \\
\text { First Trimester, } \\
\text { increase) } \\
\text { Crohn's Disease }\end{array}$ & $10,4,7$ \\
\hline
\end{tabular}

* Colors indicate different diseases or enriched taxon sets.

TABLE 3 | The detailed information of identified microbe-disease co-module 4.

\begin{tabular}{|c|c|c|c|c|}
\hline $\begin{array}{l}\text { Co- } \\
\text { module_id }\end{array}$ & $\begin{array}{l}\text { Disease } \\
\text { module }\end{array}$ & $\begin{array}{l}\text { Microbe } \\
\text { module }\end{array}$ & $\begin{array}{c}\text { Taxon sets } \\
\text { (matched disease, } \\
\text { descending order } \\
\text { by FDR) }\end{array}$ & $\begin{array}{l}\text { Associated } \\
\text { co-module }\end{array}$ \\
\hline 4 & $\begin{array}{l}\text { Allergic } \\
\text { sensitization } \\
\text { Constipation } \\
\text { IBS } \\
\text { COPD } \\
\text { Cystic } \\
\text { fibrosis } \\
\text { Eczema } \\
\text { IBD } \\
\text { New-onset } \\
\text { untreated } \\
\text { rheumatoid } \\
\text { arthrits } \\
\text { Psoriasis } \\
\text { Rheumatoid } \\
\text { arthrits } \\
\text { Ulcerative } \\
\text { colitis }\end{array}$ & $\begin{array}{l}\text { Acinetobacter } \\
\text { Bacteroides } \\
\text { ovatus } \\
\text { Bacteroides } \\
\text { vulgatus } \\
\text { Burkholderia } \\
\text { Clostridium } \\
\text { coccoides } \\
\text { Clostridium } \\
\text { difficile } \\
\text { Clostridium } \\
\text { leptum } \\
\text { Dietzia maris } \\
\text { Escherichia } \\
\text { coli } \\
\text { Lysobacter }\end{array}$ & $\begin{array}{l}\text { Cystic Fibrosis } \\
\text { Atopic dermatitis } \\
\text { Aging (decrease) } \\
\text { Dandruff } \\
\text { Crohn's Disease } \\
\text { (increase) } \\
\text { Head and neck } \\
\text { squamous cell } \\
\text { carcinoma (increase) }\end{array}$ & 9,7 \\
\hline
\end{tabular}

${ }^{*}$ Colors indicate different diseases or enriched taxon sets. 
by microbes also exist in their matched disease modules. Tables 2 and 3 show two of the identified microbe-disease co-modules and the associations between different disease (microbe) modules (according to $S_{2}$ ). As The MDNMF Algorithm shown, in trifactor NMF $X \approx H S_{H}{ }^{T}$, the matrix $S$ has a special meaning. To see this, let us assume that $H^{T} H=I$. Setting the derivative $\partial \min \| X-$ $H S_{H^{T}} \|^{2} / \partial S$ to be 0 , we can obtain:

$$
S=H^{T} X H, \text { or } S_{l k}=h_{l}^{T} X h_{k}=\frac{\sum_{i \in C_{l} \sum_{j \in C_{k}} x_{i j}}}{\sqrt{n_{l} n_{k}}} .
$$

$S$ indicates proper normalized within-cluster sum of weights $(l=k)$ and between-cluster sum of weights $(l \neq k)$. Therefore, $S$ provides a good representation for the clustering quality. If the clusters are separated well, respectively the diagonal elements of $S$ will be much larger than the off-diagonal elements. We conduct extensive experiments, and find that some off-diagonal elements are large, for example co-modules 4 and 9. According to Eq.14, this case may reflect a close connection between these two modules. The connections can provide some insights to further understand the relationships between microbe and disease, disease and disease, and microbe and microbe.

As Table 2 shown, in co-module 9, 5 of 8 diseases $(62.5 \%$, same color from disease module and taxon sets columns indicates matched or associated disease) are in accord with significantly enriched microbe TS terms (FDR < 0.05). Besides, several TS such as "Chronic Obstructive Pulmonary Disease," "Asthma," "Colorectal Carcinoma," "Resistance to Immune Checkpoint Inhibitors (increase)" which have no matched diseases are also identified. This could provide potential associations among diseases or microbes. Figure 4 shows top biological terms enriched in the microbe module 9 .
In order to demonstrate that MDNMF can indeed cluster similar diseases to the same co-module, we retrieval each disease existed in co-module 9 from the MeSH website (https://meshb. nlm.nih.gov) and find that most of the diseases belong to the same MeSH disease category. For example, Ileal Crohn's disease (CD), Irritable bowel syndrome (IBS), Liver cirrhosis and Necrotizing enterocolitis are clustered together and they are all divided into the same MeSH disease category C06 (Digestive System Diseases). Interestingly, Clostridium infections and Bacterial vaginosis which belong to $\mathrm{C} 01$ (Bacterial Infections and Mycoses) are also divided into the co-module. A detailed analysis of these related diseases may yield novel insights into the more and more widely recognized the associations between microbes and human diseases.

Based on the factorized matrix $s_{2}$, we identified the connections among microbe modules 9 and 4, 7, 10. For example, microbe modules 9 and 4 share the "Crohn's Disease" and "Head and neck squamous cell carcinoma" microbe sets, but focus opposite aspects. In microbe module 9, the enriched microbe TS term "Crohn's Disease" is decreased, but is increased in module 4 . These two microbe modules may afford us an opportunity to further investigate the complicated pathogenic mechanism in system level.

Without loss of generality, we also analyzed another microbedisease co-module 4, the detailed information is shown in Table 3.

From Table 3, we can see that 7 of 10 diseases (70\%, same color from the "disease module" and "taxon sets" columns indicates matched or associated disease) are in accord with significantly enriched microbe TS terms (FDR < 0.05). Especially, for enriched microbe TS term "Atopic dermatitis," three diseases ("Allergic sensitization," "Eczema," and "Psoriasis") in matched disease module are associated with it. This demonstrates the ability of

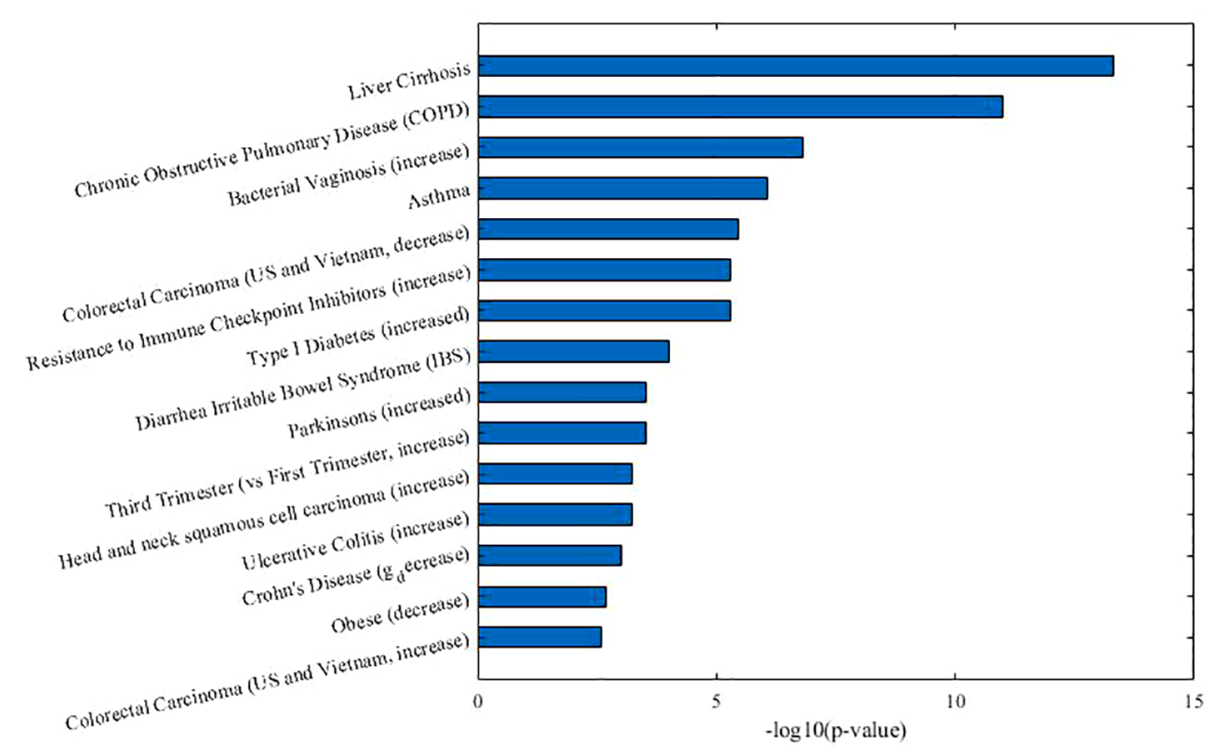

FIGURE 4 | Top enriched biological terms in microbe module 9. 


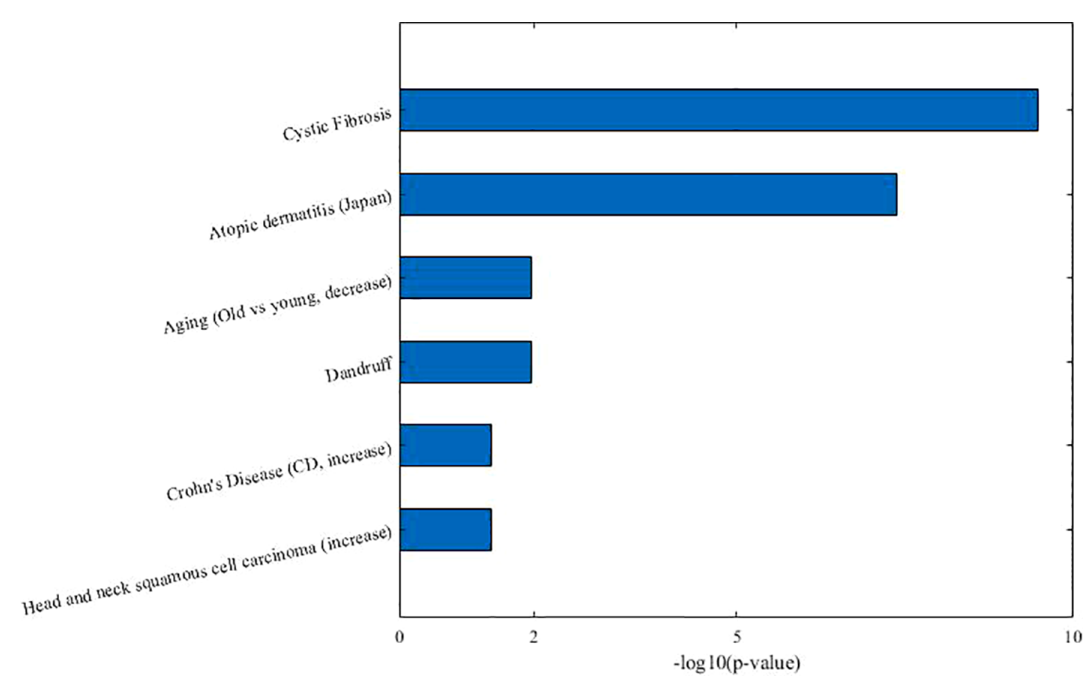

FIGURE 5 | Top enriched biological terms in microbe module 4.

the proposed MDNMF algorithm in finding correlation among diseases and microbes. Figure 5 shows top biological terms enriched in microbe module 4.

Similarly, we retrieval each disease member in co-module 4 from the MeSH website and find that a few similar diseases belong to the same $\mathrm{MeSH}$ disease category. For example, Eczema, Psoriasis, Rheumatoid arthritis, and New-onset untreated rheumatoid arthritis are all from the same MeSH disease category C17 (Skin and Connective Tissue Diseases). In addition, we also find that Chronic Obstructive Pulmonary Disease (COPD), Cystic Fibrosis, Allergic sensitization, and Intestinal diseases (IBS, Irritable bowel disease, and Ulcerative colitis) have also been clustered together. Several diseases belong to two or more $\mathrm{MeSH}$ categories, which indicates the pathological connections between the human genetic susceptibility to infectious diseases and inflammatory diseases.

Based on factorized matrix $s_{2}$, we can find that co-module 4 has more links to co-module $7\left(s_{4.7}=2.72\right)$. Matched disease modules 4 and 7 own the similar disease members, such as "Allergic sensitization" (from module 4) and "Asthma" (from module 7) induced by "Atopic dermatitis." Besides, two corresponding microbe modules 4 and 7 share TS term "Aging."

Note that in Tables $\mathbf{2}$ and $\mathbf{3}$ some related diseases and microbes are divided into different co-modules. One possible of reasons is that the connection weight between these comodules is large, MDNMF as a soft clustering approach, cannot well separately these related microbes or disease. In the future, we will design more robust threshold selecting method to assign each diseases or microbes to accurate modules.

In summary, for the identified module pairs by MDNMF, especially for microbe modules, some of them share a few biological functions (TS), but also have their special roles. Simultaneously, some associations between microbe modules, disease modules can be also detected by MDNMF.

\section{CONCLUSIONS}

The association between microbes and human diseases has been verified by more and more researches. However, previous studies mainly focused on detecting the relationship such as "one microbe, one disease," rarely analyzed the pathogenesis of microbial-related complex diseases from a modular perspective. In this paper, we propose a novel microbe-disease co-module detecting algorithm MDNMF to construct a two-level module network by integrating two similarity matrices (microbe-microbe, disease-disease similarity matrices) and one microbe-disease bipartite network. Using the identified individual modules from different levels (microbe, disease levels) and their links, we are able to find a few disease-related microbes (taxon sets) which provide an opportunity to further understand the microbe high-order relationship and their potential functions.

Meanwhile, in order to improve the accuracy of module finding and biological interpretability of modules identified by MDNMF, we introduce human symptoms-disease network and microbial phylogenetic distance into the model. Compared with other two NMF-based approaches, MDNMF can achieve better performance in terms of $E I$ and the number of significantly enriched taxon sets. The proposed MDNMF is also easily extended to other multiple-level molecular network application, for example, virus-host co-modules, microbe-drug co-modules discovering, and so on.

\section{DATA AVAILABILITY STATEMENT}

The data and MDNMF codes analyzed during the study are available in the GitHub repository, https://github.com/ chonghua-1983/MDNMF. 


\section{AUTHOR CONTRIBUTIONS}

YuM wrote the manuscript and developed the algorithms. YuM and GL developed the concept for the structure and content of the manuscript. YiM wrote the code used in the paper. QC critically revised the manuscript in final. All authors reviewed and approved the final version of the manuscript.

\section{REFERENCES}

Barabasi, A.-L., and Oltvai, Z. N. (2004). Network biology: understanding the cell's functional organization. Nat. Rev. Genet. 5, 101-113. doi: 10.1038/nrg1272

Blaser, M. (2014). Missing microbes (United Kingdom and Commonwealth: Oneworld Publications).

Boleij, A., Hechenbleikner, E. M., Goodwin, A. C., Badani, R., Stein, E. M., Lazarev, M. G., et al. (2014). The Bacteroides fragilis toxin gene is prevalent in the colon mucosa of colorectal cancer patients. Clin. Infect. Dis. 60, 208-215. doi: $10.1093 / \mathrm{cid} / \mathrm{ciu} 787$

Cai, J., Cai, H., Chen, J., and Yang, X. (2018). Identifying "Many-to-Many" relationships between gene-expression data and drug-response data via sparse binary matching. IEEE/ACM Trans. Comput. Biol. Bioinf. doi: 10.1109/ TCBB.2018.2849708

Chen, Y., and Blaser, M. J. (2007). Inverse associations of Helicobacter pylori with asthma and allergy. Arch. Internal Med. 167, 821-827. doi: 10.1001/ archinte.167.8.821

Chen, J., and Zhang, S. (2016). Integrative analysis for identifying joint modular patterns of gene-expression and drug-response data. Bioinformatics 32, 17241732. doi: 10.1093/bioinformatics/btw059

Chen, J., and Zhang, S. (2018). Discovery of two-level modular organization from matched genomic data via joint matrix tri-factorization. Nucleic Acids Res. 46, 5967-5976. doi: 10.1093/nar/gky440

Chen, J., Peng, H., Han, G., Cai, H., and Cai, J. (2018). HOGMMNC: a higher order graph matching with multiple network constraints model for gene-drug regulatory modules identification. Bioinformatics 35, 602-610. doi: 10.1093/ bioinformatics/bty662

Dhariwal, A., Chong, J., Habib, S., King, I. L., Agellon, L. B., and Xia, J. (2017). MicrobiomeAnalyst: a web-based tool for comprehensive statistical, visual and meta-analysis of microbiome data. Nucleic Acids Res. 45, W180-W188. doi: 10.1093/nar/gkx295

Ding, C., He, X., and Simon, H. D. (2005). "On the equivalence of nonnegative matrix factorization and spectral clustering," in Proceedings of the 2005 SIAM International Conference on Data Mining (SIAM), USA, 606-610.

Ding, C., Li, T., Peng, W., and Park, H. (2006). "Orthogonal nonnegative matrix t-factorizations for clustering," in Proceedings of the 12th ACM SIGKDD international conference on Knowledge discovery and data mining (ACM), New York, NY, 126-135.

Ehrlich, S. D., and Consortium, M. (2011). "MetaHIT: The European Union Project on metagenomics of the human intestinal tract," in Metagenomics of the human body. (New York, NY: Springer), 307-316.

Fredricks, D. N., Fiedler, T. L., and Marrazzo, J. M. (2005). Molecular identification of bacteria associated with bacterial vaginosis. New Engl. J. Med. 353, 1899-1911. doi: 10.1056/NEJMoa043802

Gevers, D., Knight, R., Petrosino, J. F., Huang, K., Mcguire, A. L., Birren, B. W., et al. (2012). The Human microbiome project: a community resource for the healthy human microbiome. PloS Biol. 10, e1001377. doi: 10.1371/journal.pbio.1001377

Gilbert, J. A., Meyer, F., Antonopoulos, D., Balaji, P., Brown, C. T., Brown, C. T., et al. (2010). Meeting report: the terabase metagenomics workshop and the vision of an Earth microbiome project. Standards In Genomic Sci. 3, 243-248. doi: 10.4056/sigs. 1433550

Guo, X., and Boedicker, J. Q. (2016). The contribution of high-order metabolic interactions to the global activity of a four-species microbial community. PloS Comput. Biol. 12, e1005079. doi: 10.1371/journal.pcbi.1005079

\section{FUNDING}

This work has been accepted by CBC2019, supported by the National Natural Science Foundation of China (No.61532008), Key Research Projects of Henan Higher Education Institutions (No. 20B520002), The Key Technology R \& D Program of Henan Province (202102310561)

He, B.-S., Peng, L.-H., and Li, Z. (2018). Human microbe-disease association prediction with graph regularized non-negative matrix factorization. Front. Microbiol. 9. doi: 10.3389/fmicb.2018.02560

Huang, Y.-A., You, Z.-H., Chen, X., Huang, Z.-A., Zhang, S., and Yan, G.-Y. (2017). Prediction of microbe-disease association from the integration of neighbor and graph with collaborative recommendation model. J. Trans. Med. 15, 209. doi: 10.1186/s12967-017-1304-7

Jorth, P., Turner, K. H., Gumus, P., Nizam, N., Buduneli, N., and Whiteley, M. (2014). Metatranscriptomics of the human oral microbiome during health and disease. MBio 5, e01012-e01014. doi: 10.1128/mBio.01012-14

Kutalik, Z., Beckmann, J. S., and Bergmann, S. (2008). A modular approach for integrative analysis of large-scale gene-expression and drug-response data. Nat. Biotechnol. 26, 531-539. doi: 10.1038/nbt1397

Ma, W., Zhang, L., Zeng, P., Huang, C., Li, J., Geng, B., et al. (2016a). An analysis of human microbe-disease associations. Brief. Bioinf. 18, 85-97. doi: 10.1093/ bib/bbw005

Ma, Y., Hu, X., He, T., and Jiang, X. (2016b). Hessian regularization based symmetric nonnegative matrix factorization for clustering gene expression and microbiome data. Methods 111, 80-84. doi: 10.1016/j.ymeth. 2016.06.017

Ma, Y., Hu, X., He, T., and Jiang, X. (2017). Clustering and integrating of heterogeneous microbiome data by joint symmetric nonnegative matrix factorization with laplacian regularization. IEEE/ACM Trans. Comput. Biol. Bioinf. doi: 10.1109/TCBB.2017.2756628

Medzhitov, R. (2007). Recognition of microorganisms and activation of the immune response. Nature 449, 819-826. doi: 10.1038/nature06246

Micah, H., Claire, F.-L., and Rob, K. (2007). The human microbiome project: exploring the microbial part of ourselves in a changing world. Nature 449, 804810. doi: $10.1038 /$ nature 06244

Qin, J., Li, R., Raes, J., Arumugam, M., Burgdorf, K. S., Manichanh, C., et al. (2010). A human gut microbial gene catalogue established by metagenomic sequencing. Nature 464, 59-65. doi: 10.1038/nature08821

Shah, P., Fritz, J. V., Glaab, E., Desai, M. S., Greenhalgh, K., Frachet, A., et al. (2016). A microfluidics-based in vitro model of the gastrointestinal humanmicrobe interface. Nat. Commun. 7, 11535. doi: 10.1038/ncomms11535

Socransky, S., Haffajee, A., Cugini, M., Smith, C., and Kent, R.Jr. (1998). Microbial complexes in subgingival plaque. J. Clin. Periodontol. 25, 134-144. doi: 10.1111/j.1600-051X.1998.tb02419.x

Thiele, I., Heinken, A., and Fleming, R. M. (2013). A systems biology approach to studying the role of microbes in human health. Curr. Opin. Biotechnol. 24, 412. doi: 10.1016/j.copbio.2012.10.001

Turnbaugh, P. J., Hamady, M., Yatsunenko, T., Cantarel, B. L., Duncan, A., Ley, R. E., et al. (2009). A core gut microbiome in obese and lean twins. Nature 457, 480-484. doi: 10.1038/nature07540

Van Dam, S., Vosa, U., Van Der Graaf, A., Franke, L., and De Magalhaes, J. P. (2017). Gene co-expression analysis for functional classification and genedisease predictions. Brief. Bioinf. 19, 575-592. doi: 10.1093/bib/bbw139

Wang, F., Huang, Z.-A., Chen, X., Zhu, Z., Wen, Z., Zhao, J., et al. (2017). LRLSHMDA: laplacian regularized least squares for human microbedisease association prediction. Sci. Rep. 7, 7601. doi: 10.1038/s41598-01708127-2

Zhang, W., Yang, W., Lu, X., Huang, F., and Luo, F. (2018a). The bi-direction similarity integration method for predicting microbe-disease associations. IEEE Access 6, 38052-38061. doi: 10.1109/ACCESS.2018.2851751 
Zhang, W., Yue, X., Tang, G., Wu, W., Huang, F., and Zhang, X. (2018b). SFPELLPI: Sequence-based feature projection ensemble learning for predicting LncRNA-protein interactions. PloS Comput. Biol. 14, e1006616. doi: 10.1371/ journal.pcbi.1006616

Zhang, W., Jing, K., Huang, F., Chen, Y., Li, B., Li, J., et al. (2019). SFLLN: A sparse feature learning ensemble method with linear neighborhood regularization for predicting drug-drug interactions. Inf. Sci. 497, 189-201. doi: 10.1016/ j.ins.2019.05.017

Zhou, X., Menche, J., Barabási, A.-L., and Sharma, A. (2014). Human symptomsdisease network. Nat. Commun. 5, 4212. doi: 10.1038/ncomms5212
Conflict of Interest: The authors declare that the research was conducted in the absence of any commercial or financial relationships that could be construed as a potential conflict of interest.

Copyright (c) $2020 \mathrm{Ma}, \mathrm{Liu}, \mathrm{Ma}$ and Chen. This is an open-access article distributed under the terms of the Creative Commons Attribution License (CC BY). The use, distribution or reproduction in other forums is permitted, provided the original author (s) and the copyright owner(s) are credited and that the original publication in this journal is cited, in accordance with accepted academic practice. No use, distribution or reproduction is permitted which does not comply with these terms. 